\title{
Human Mars Entry, Descent, and Landing Architecture Study: Phase 3 Summary
}

Damien Calderon

Holly Newton

Joseph Amar

Zachery Wiens

NASA Johnson Space Center

\section{Joseph A. Garcia}

NASA Ames Research Center

AIAA Science and Technology Forum | 2020 January 6-10 /Orlando, FL 


\title{
Contents
}

\section{Project Background}

\author{
Outer Mold Line Update
}

Manifest Packaging Study

Structural Sizing and Optimization 
Joseph Garcia

OUTER MOLD LINE UPDATE 


\section{Background}

- Mid Lift-to-Drag (Mid L/D) vehicle being considered by NASA to land large payloads on the surface of Mars

- During Entry, Descent, and Landing Architecture Study (EDLAS) phase 3, Mid L/D conceptual design advanced significantly

- Updated outer mold line (OML) shape

- Conducted packaging feasibility study

- Completed preliminary vehicle structure design and mass sizing 


\section{CobraMRV OML Update}

- Co-Optimization Blunt-body Re-entry Analysis (COBRA) has been used by NASA for the past decade to arrive at a shape for the Mid L/D rigid vehicle (MRV)

- Goal to minimize surface heating, maximize drag area $\left(C_{d} A\right)$

- Realistic body flap concept allowed integration of OML with body flap shape 


\section{COBRA Optimization}

- Pareto front of multi-objective genetic algorithm (MOGA) shapes (left) used to down select CobraMRV2908 shape (right)
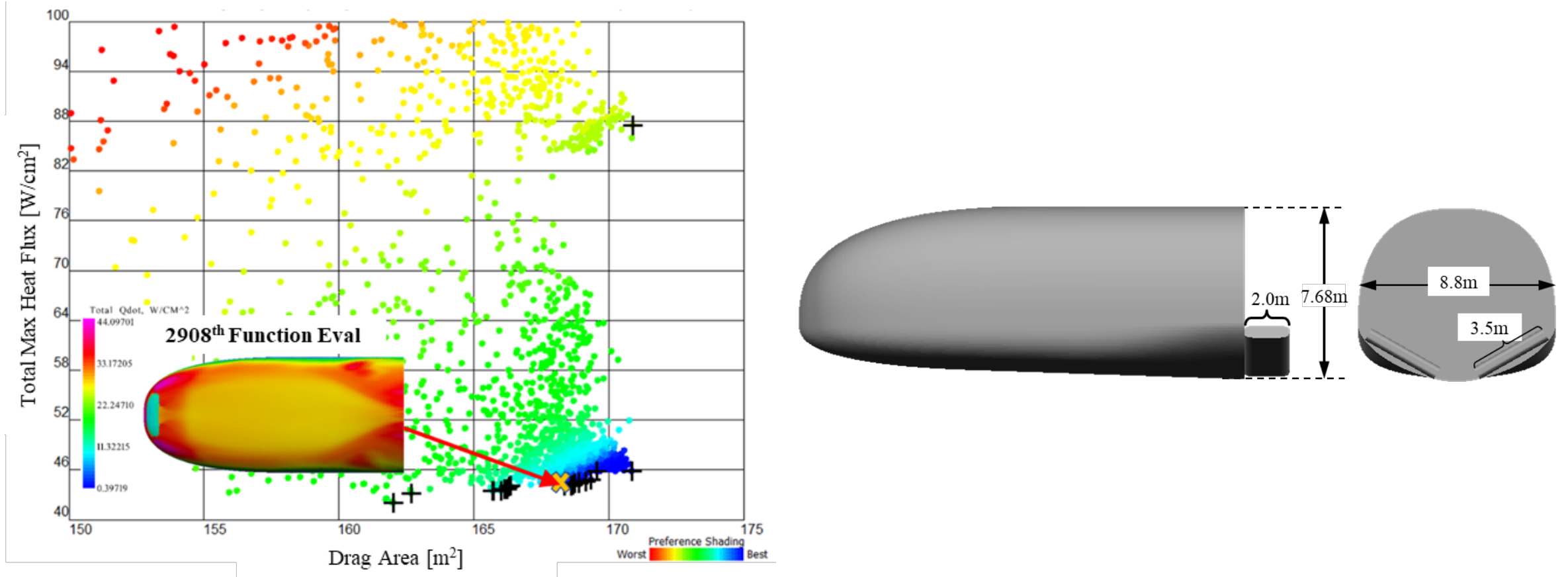


\section{COBRA Optimization}

\section{- 2908 shape was modified to 2908 b}

- Allowed fit within SLS 10m shroud (Ø9.1 m inner volume)

- Reduced high heating and flow separation on body flaps

2908
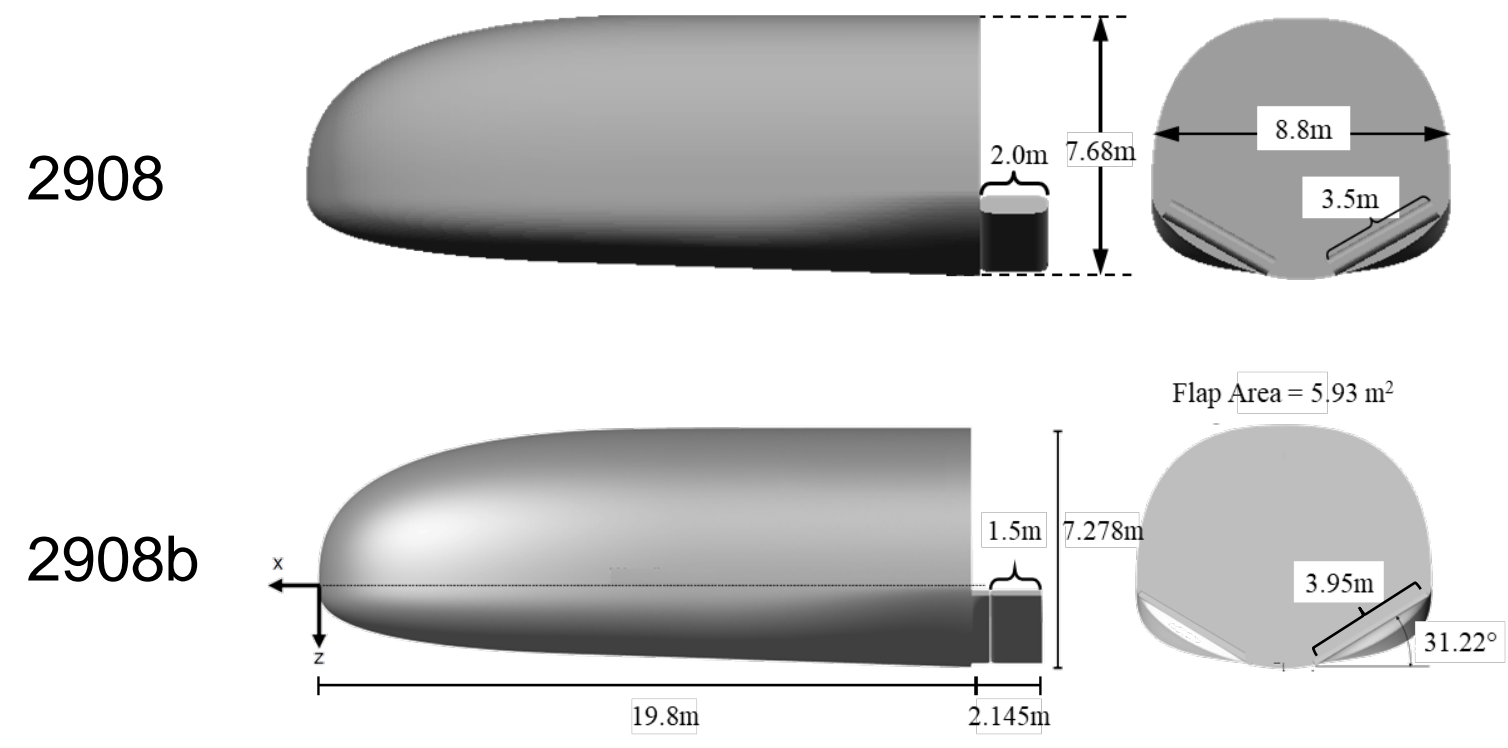


\section{Body Flap Concept}

- Conceptual design for body flap structure and actuators enabled more realistic modelling

- Below concept was based on Space Shuttle Orbiter design

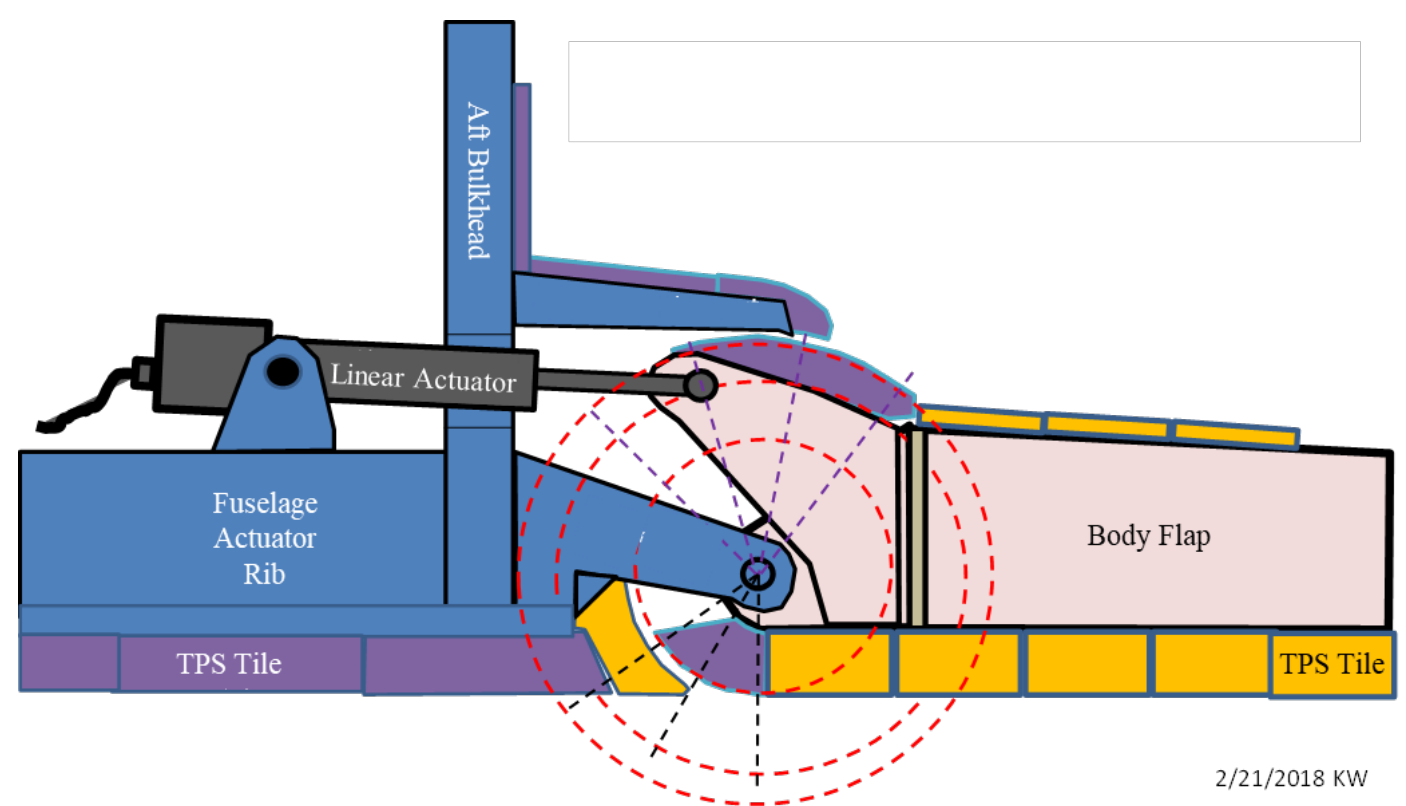




\section{COBRA Iteration}

- $2908 \mathrm{c} \rightarrow \mathbf{2 9 0 8 d} \rightarrow \mathbf{2 9 0 8 \mathrm { e }} \rightarrow \mathbf{2 9 0 8 \mathrm { f }} \rightarrow \mathbf{2 9 0 8 g}$

- Re-lofted aft $2.5 \mathrm{~m}$ of OML to smooth body flap transition

- Changed to trapezoidal flaps

- Increased cant angle and planform area of flaps

- Increased aft body base corner radii to reduce heating

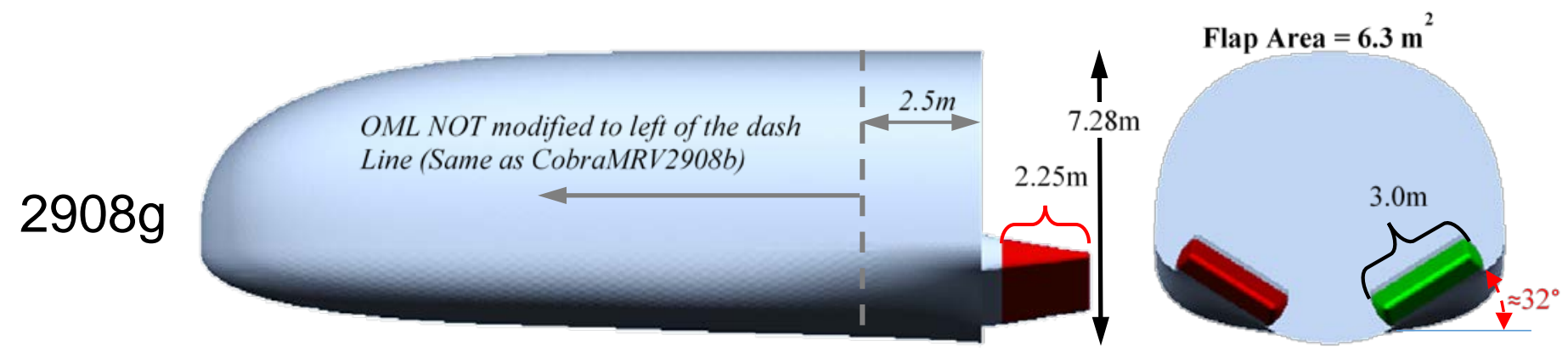




\section{8g Comparison}

- Used CBAERO Newtonian aerodynamic tool to compare $2908 \mathrm{~g}$ to $2908 \mathrm{~b}$

- Similar Coefficient of Lift

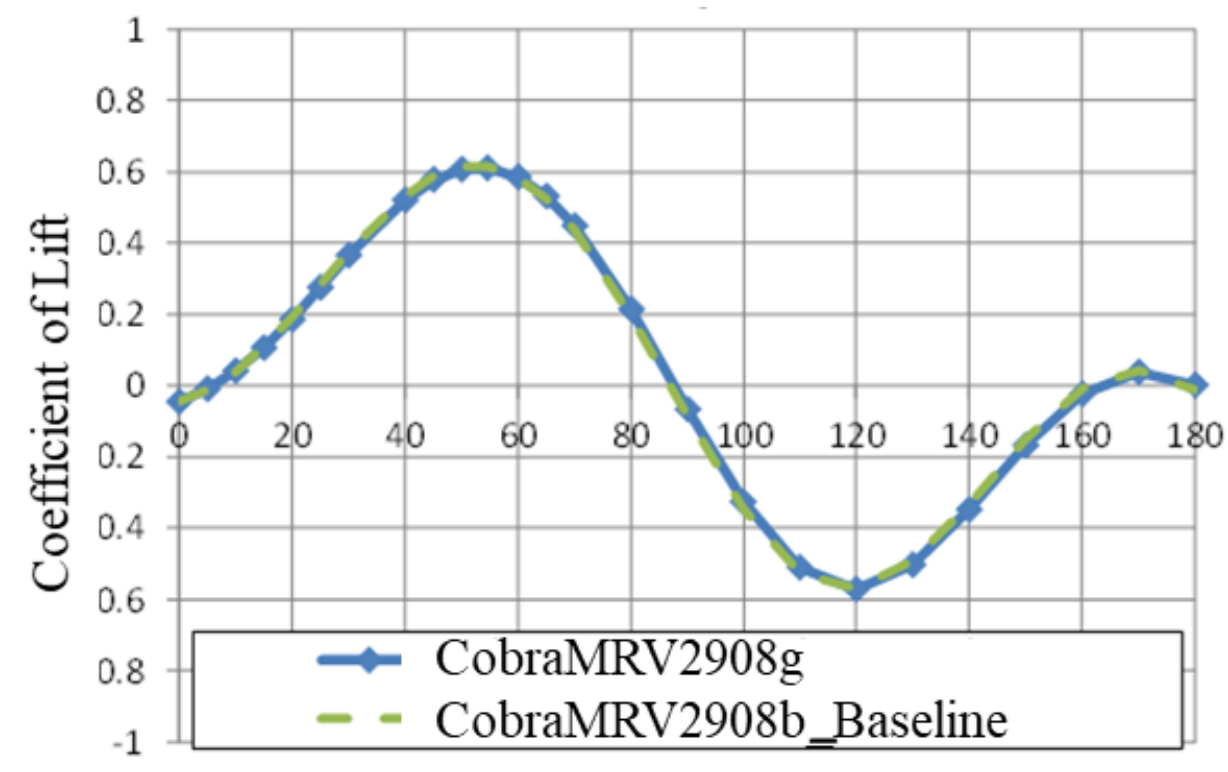

Alpha (deg) 


\section{8g Comparison}

- Used CBAERO Newtonian aerodynamic tool to compare $2908 \mathrm{~g}$ to $2908 \mathrm{~b}$

- Similar Coefficient of Drag

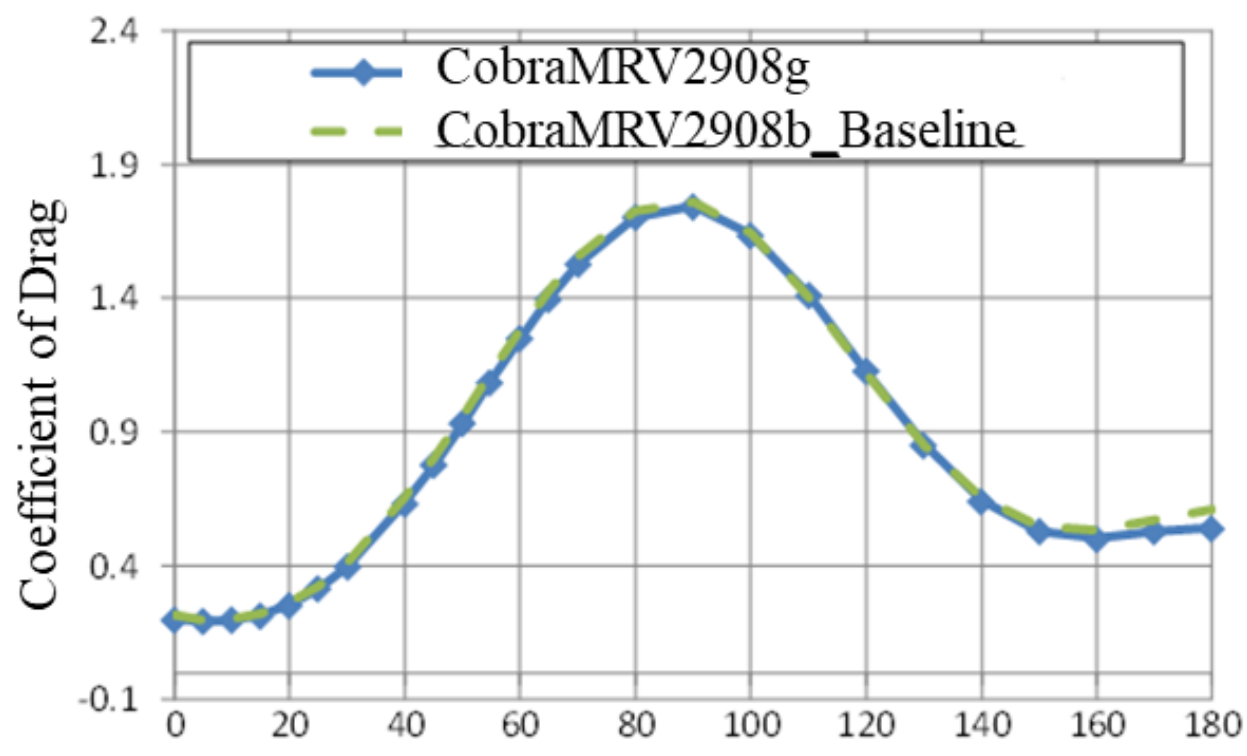

Alpha (deg) 


\section{8g Comparison}

- Used CBAERO Newtonian aerodynamic tool to compare $2908 \mathrm{~g}$ to $2908 \mathrm{~b}$

- Improved pitching moment coefficient at high angles of attack

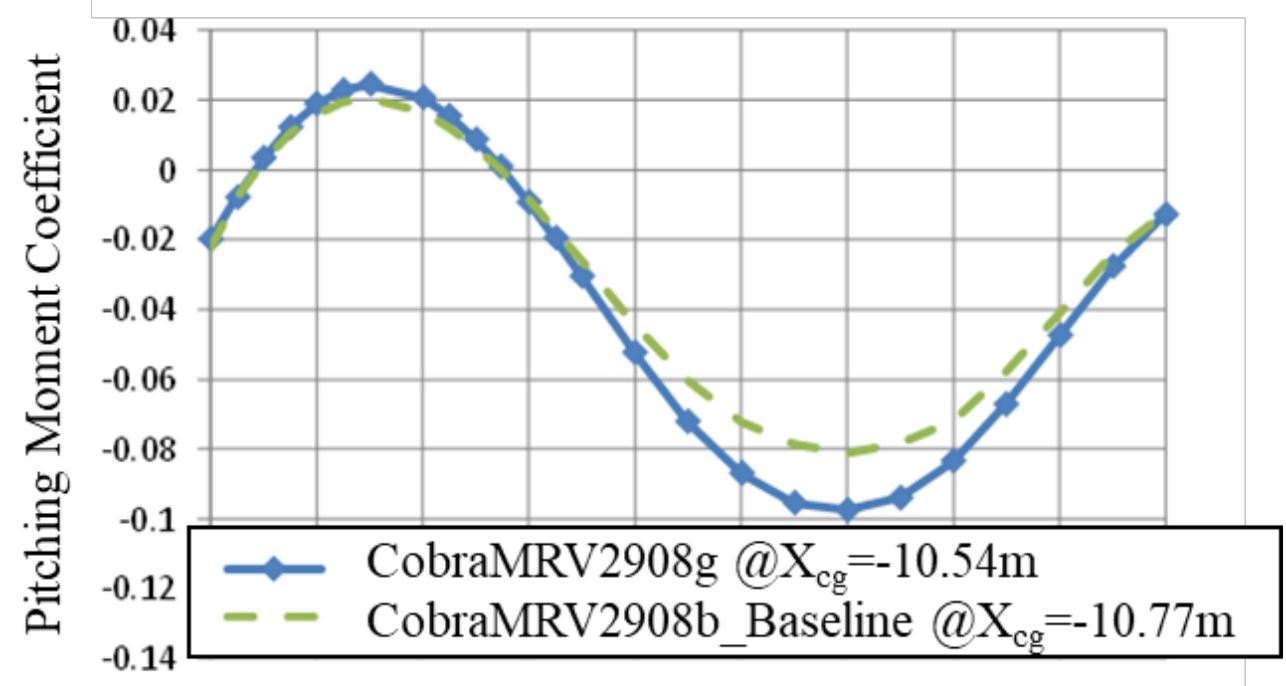

Alpha (deg) 


\section{8g Comparison}

- Used Kestrel computational fluid dynamics software to verify flow improvements

- Improved flow onto body flaps

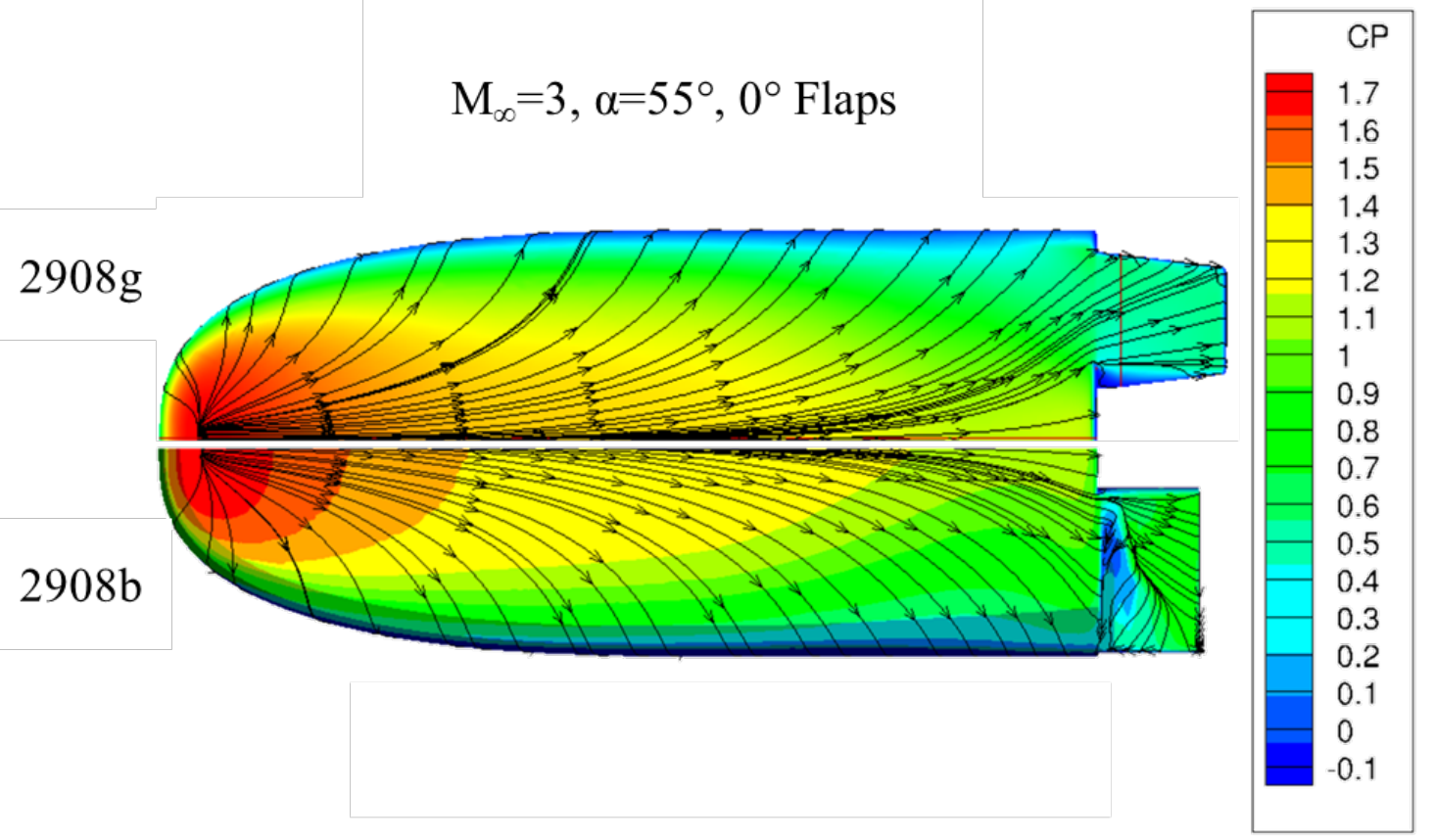


Joseph Amar, Holly Newton, Zachery Wiens MANIFEST PACKAGING 


\section{Assumptions}

- Assumed 22T Payload manifest for Crew Expedition 1 Mission

-Cargo-1, Cargo-2, Crew-1

-Common items across manifest would be identically placed

- Used the 2908G OML, manifest packaging for Cargo 2 for sizing

-Modeled frame to match OML form

- Creo Parametric software used to conuct packaging and CG estimation 


\section{CG Requirement}

- CG should be mid-body of MRV, along launch vehicle (LV) axis

\begin{tabular}{|c|r|r|}
\hline Axis & Minimum $(\mathbf{m})$ & \multicolumn{1}{|c|}{ Maximum (m) } \\
\hline$X$ & -10.747 & -10.347 \\
\hline$Y$ & -0.050 & 0.050 \\
\hline$Z$ & -1.100 & 0.000 \\
\hline
\end{tabular}

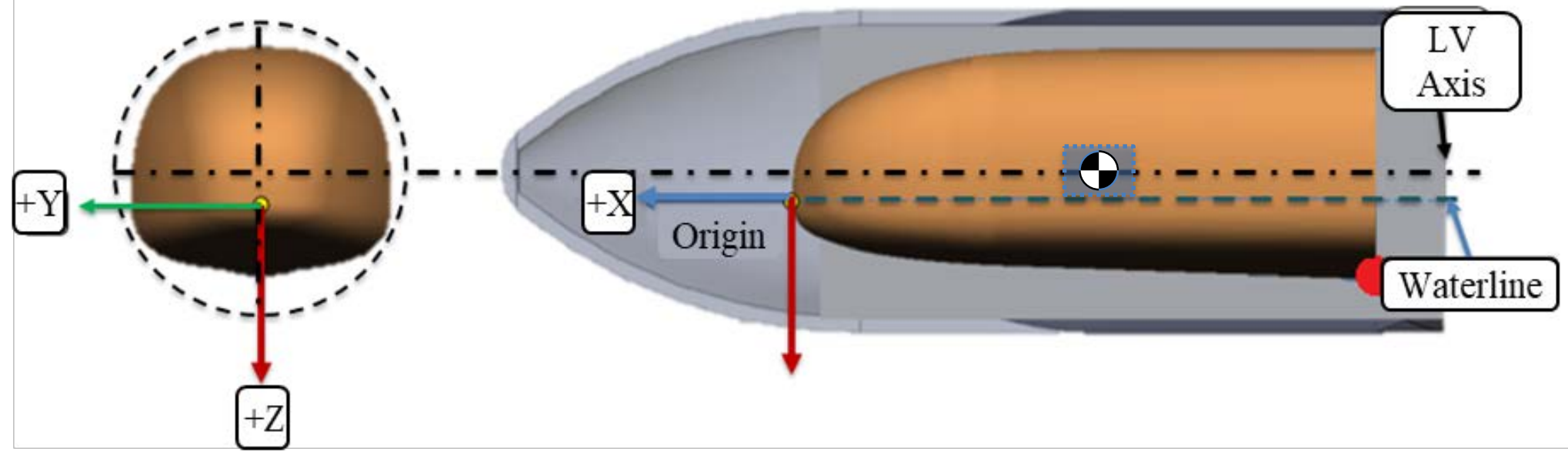




\section{Expedition 1 Common Subsystems}

\begin{tabular}{|c|c|c|c|}
\hline Component & Qty. & $(\mathrm{kg}) \mathrm{N}$ & s Total \\
\hline Thermal Protection System (TPS) & & & 4624 \\
\hline Windward & 1 & 3590 & 3590.21 \\
\hline Aft Bulkhead & 1 & 65 & 64.75 \\
\hline Aft Door & 1 & 73 & 73.40 \\
\hline Leeward & 1 & 653 & 653.13 \\
\hline Cargo Bay Doors & 2 & 121 & 242.50 \\
\hline Acoustic Thermal Protection - Dist. & & & 635 \\
\hline IML Blankets & 1 & 459 & 458.80 \\
\hline IML Radiant Barrier - MLI & 1 & 65 & 65.10 \\
\hline Lightning Protection & 1 & 111 & 110.90 \\
\hline Power & & & 470.00 \\
\hline Power Distribution & 1 & 400 & 400.00 \\
\hline Power Cable (1 km) spool & 1 & 70 & 70.00 \\
\hline Body Flaps & 2 & 298 & 595.40 \\
\hline Landing Gear & 3 & 388 & 1164.60 \\
\hline Avionics & & & 333.00 \\
\hline Command and Data Handling & 1 & 141 & 141.00 \\
\hline Communications and Tracking & 1 & 76 & 76.00 \\
\hline Guidance, Navigation, \& Control & 1 & 116 & 116.00 \\
\hline Environmental Cabin & & & 212.50 \\
\hline Active Cooling Loops & 1 & 200 & 200.00 \\
\hline Heaters & 1 & 13 & 12.50 \\
\hline
\end{tabular}

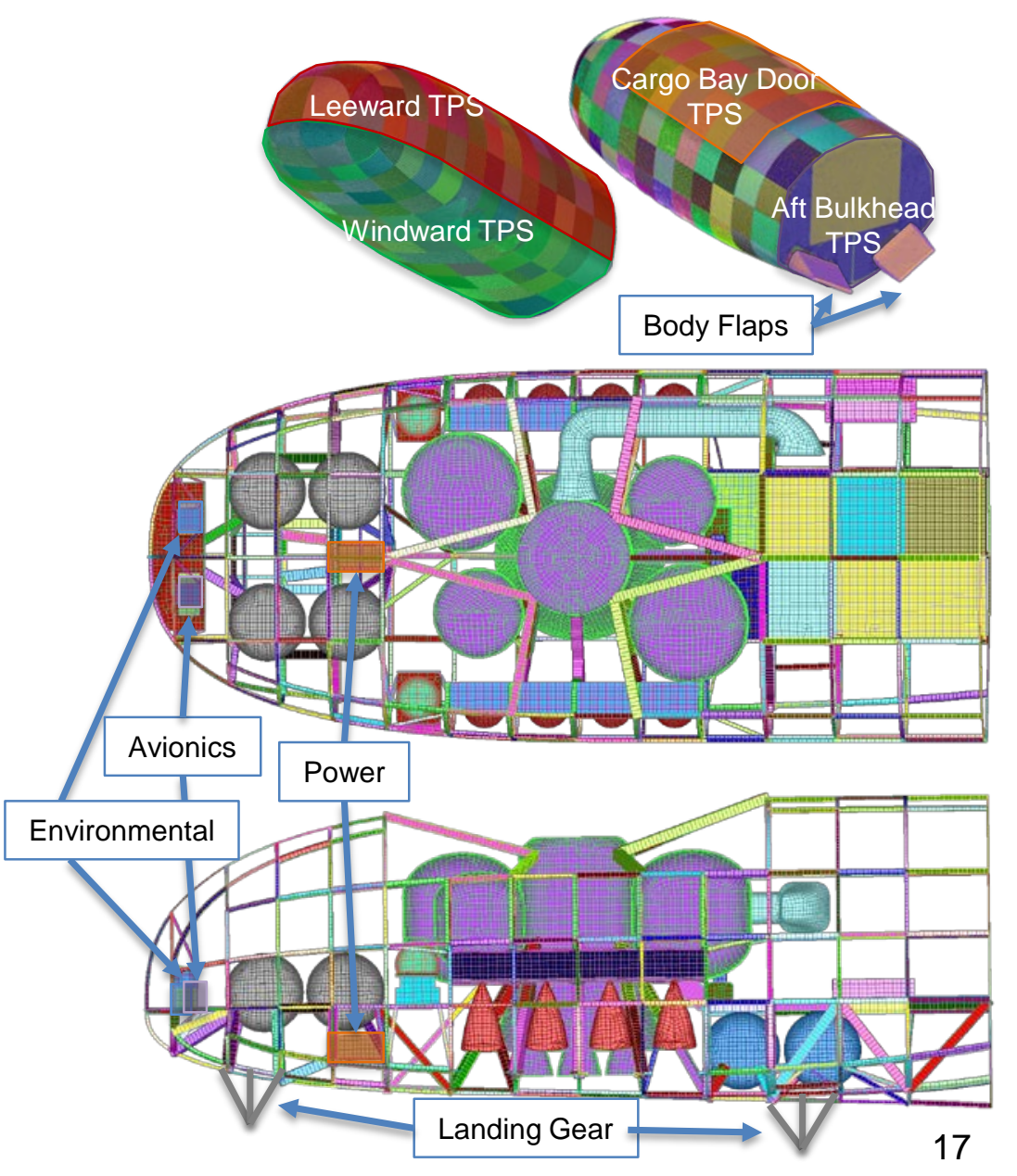




\section{Expedition 1 Common Subsystems}

\begin{tabular}{|c|c|c|c|}
\hline Component & Qty. & $\mathrm{kg})$ & Total \\
\hline In-Space Radiator & 1 & 191 & 191 \\
\hline Other - Dist. & & & 379 \\
\hline Purge System & 1 & 301 & 301 \\
\hline Umbilicals Interfaces & 1 & 78 & 78 \\
\hline Methane + Tank & 4 & 1080 & 4322 \\
\hline LOX + Tank & 4 & 3274 & 13095 \\
\hline Fuel Subsystem & 2 & 328 & 656 \\
\hline RCS Prop Distribution & 2 & 205 & 411 \\
\hline RCS Cluster & 2 & 506 & 1013 \\
\hline Main Engine & 8 & 250 & 2000 \\
\hline Aft Ramp System & & & 469 \\
\hline Strut & 2 & 8 & 16 \\
\hline Hard stop & 1 & 49 & 49 \\
\hline Hinges & 1 & 22 & 22 \\
\hline Perimeter Seal & 1 & 41 & 41 \\
\hline Ramp & 1 & 175 & 175 \\
\hline Mechanism & 1 & 166 & 166 \\
\hline Cargo Bay Doors Mech. & & & 360 \\
\hline Power Drive Units & 10 & 10 & 96 \\
\hline Rotary Actuators & 8 & 6 & 46 \\
\hline Latches & 22 & 8 & 168 \\
\hline Hinges & 16 & 3 & 50 \\
\hline
\end{tabular}

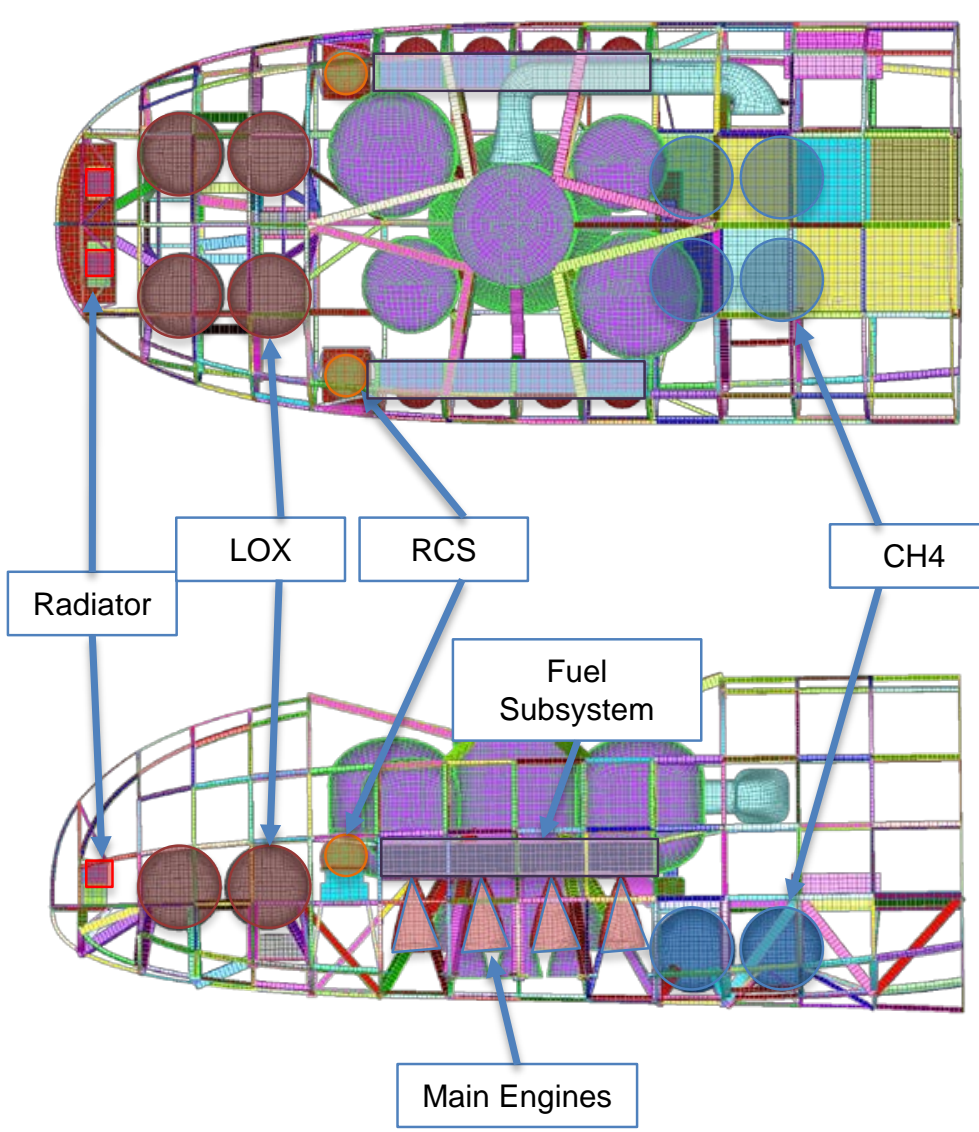




\section{Expedition 1 Payload Manifest}

\section{- Cargo-1}

\begin{tabular}{|l|c|c|c|}
\hline Manifested Item & Mass (kg) & Qty & Mass (kg) \\
\hline Kilopower, 10 kW each & 1544 & 5 & 7720 \\
\hline Power Management/Distribution & 400 & 1 & 400 \\
\hline Power cable (1 km) spool & 70 & 1 & 70 \\
\hline Crew Support Rover & 1225 & 1 & 1225 \\
\hline Cargo Hoist & 600 & 1 & 600 \\
\hline Crew Descent Module & 3516 & 1 & 3516 \\
\hline Mars Mobility Chassis & 2457 & 1 & 2457 \\
\hline Pressurized Rover & 6021 & 1 & 6021 \\
\hline Total Payload Mass & & 22009 \\
\hline
\end{tabular}




\section{Cargo-1 Packaging}

\section{Zachery Wiens}

Mars Mobility
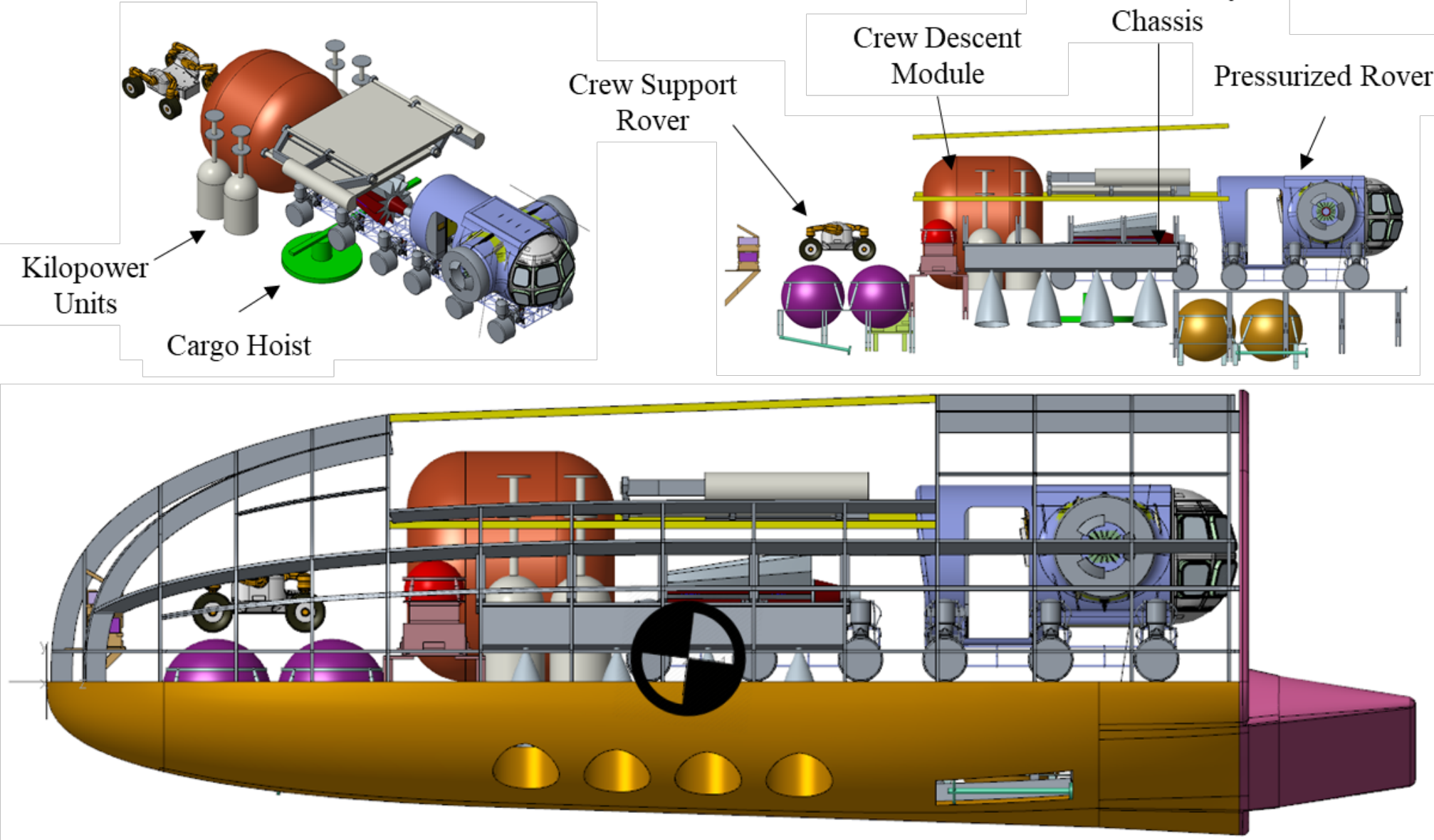


\section{Expedition 1 Payload Manifest}

\section{- Cargo-2}

\begin{tabular}{|l|c|c|c|}
\hline Manifested Item & Mass (kg) & Qty & Mass (kg) \\
\hline Atmospheric Production Plant & 1032 & 1 & 1032 \\
\hline Part of ISRU radiator mass & 478 & 1 & 478 \\
\hline ISRU Deployment & 130 & 1 & 130 \\
\hline Power Management/Distribution & 400 & 1 & 400 \\
\hline Power cable (1 km) spool & 70 & 1 & 70 \\
\hline Connecting Tunnel & 237 & 1 & 237 \\
\hline Allocated Science Payload & 1000 & 0.2 & 200 \\
\hline MAV (5 sol, wet) & 18868 & 1 & 18868 \\
\hline Part of MAV Radiator & 212 & 1 & 212 \\
\hline MPS Tank Cryocoolers/BAC charged to MAV & 141 & 1 & 141 \\
\hline MDM-to-MAV Adapter & 200 & 1 & 200 \\
\hline Total Payload Mass & & 21968 \\
\hline
\end{tabular}




\section{Cargo-2 Packaging}

Holly Newton
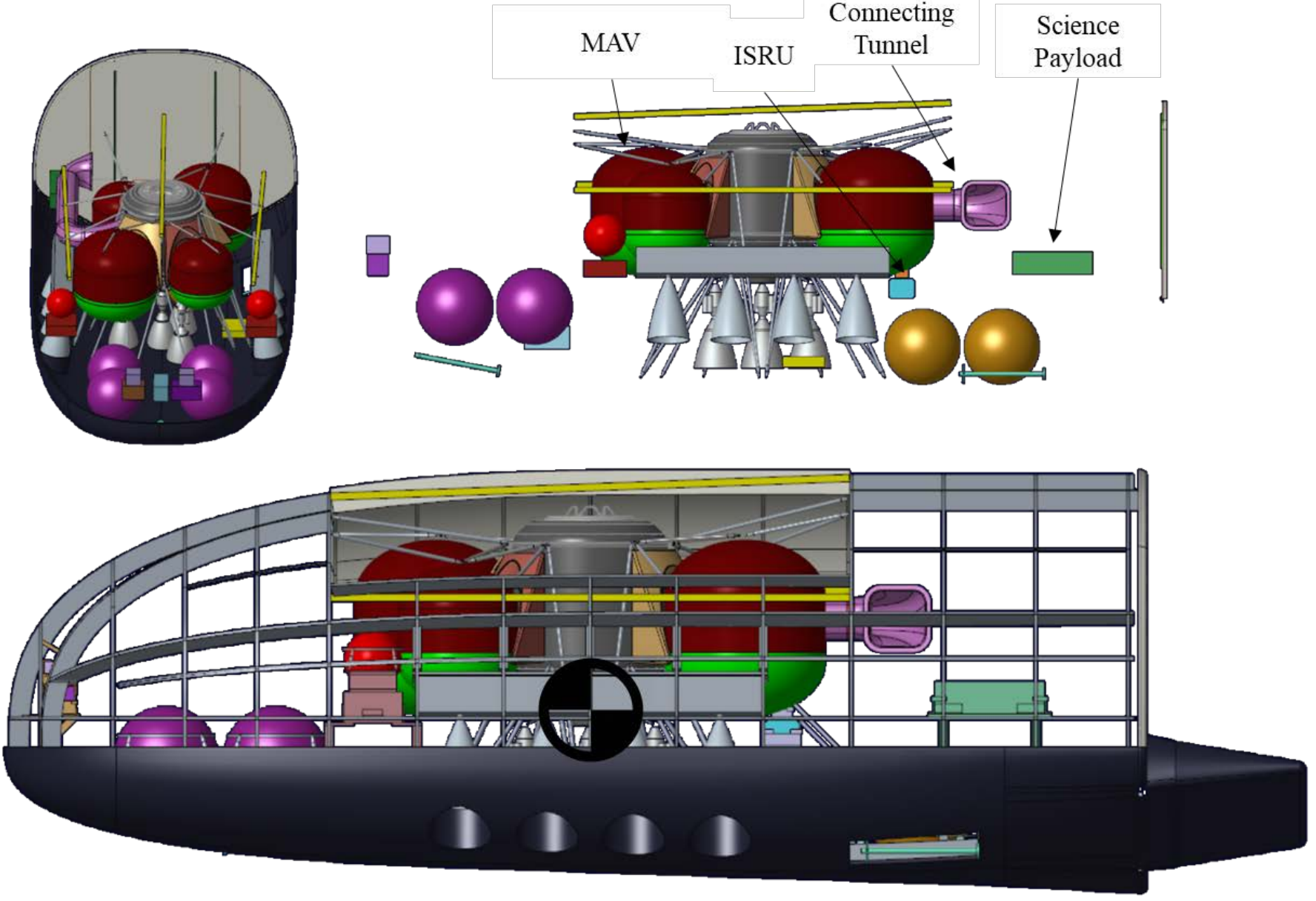


\section{Expedition 1 Payload Manifest}

\section{- Crew-1}

\begin{tabular}{|l|c|c|c|}
\hline Manifested Item & Mass (kg) & Qty & Mass (kg) \\
\hline Crew (each) & 100 & 4 & 400 \\
\hline Power Management/Distribution & 400 & 1 & 400 \\
\hline Power cable (1 km) spool & 70 & 1 & 70 \\
\hline Cargo Hoist & 600 & 1 & 600 \\
\hline Logistics Module (Dual Hatch 3500 kg capacity) & 2600 & 2 & 5200 \\
\hline Crew Descent Module & 3516 & 1 & 3516 \\
\hline Airlock Module & 3500 & 1 & 3500 \\
\hline Connecting Tunnel & 237 & 1 & 237 \\
\hline Consumables (4.02 kg/person/sol + 97.57) & 4.02 & 163 & 2719 \\
\hline Spares and Other Logistics (2.946 kg/sol + 2112.9 kg) & 2.946 & 163 & 2593 \\
\hline Maintenance Equipment & 70 & 1 & 70 \\
\hline EVA Suit + Primary Life Support System & 693 & 1 & 693 \\
\hline Launch-Entry Assembly Suits & 104 & 1 & 104 \\
\hline Spares (1.73 kg/sol/crew + 796.8) & 4 crew & 163 & 1079 \\
\hline Allocated Science Payload & 1000 & 0.8 & 800 \\
\hline & & 21981 \\
\hline
\end{tabular}




\section{Crew-1 Packaging}

\section{Joseph Amar}

Airlock Module Logistics Module (x2)
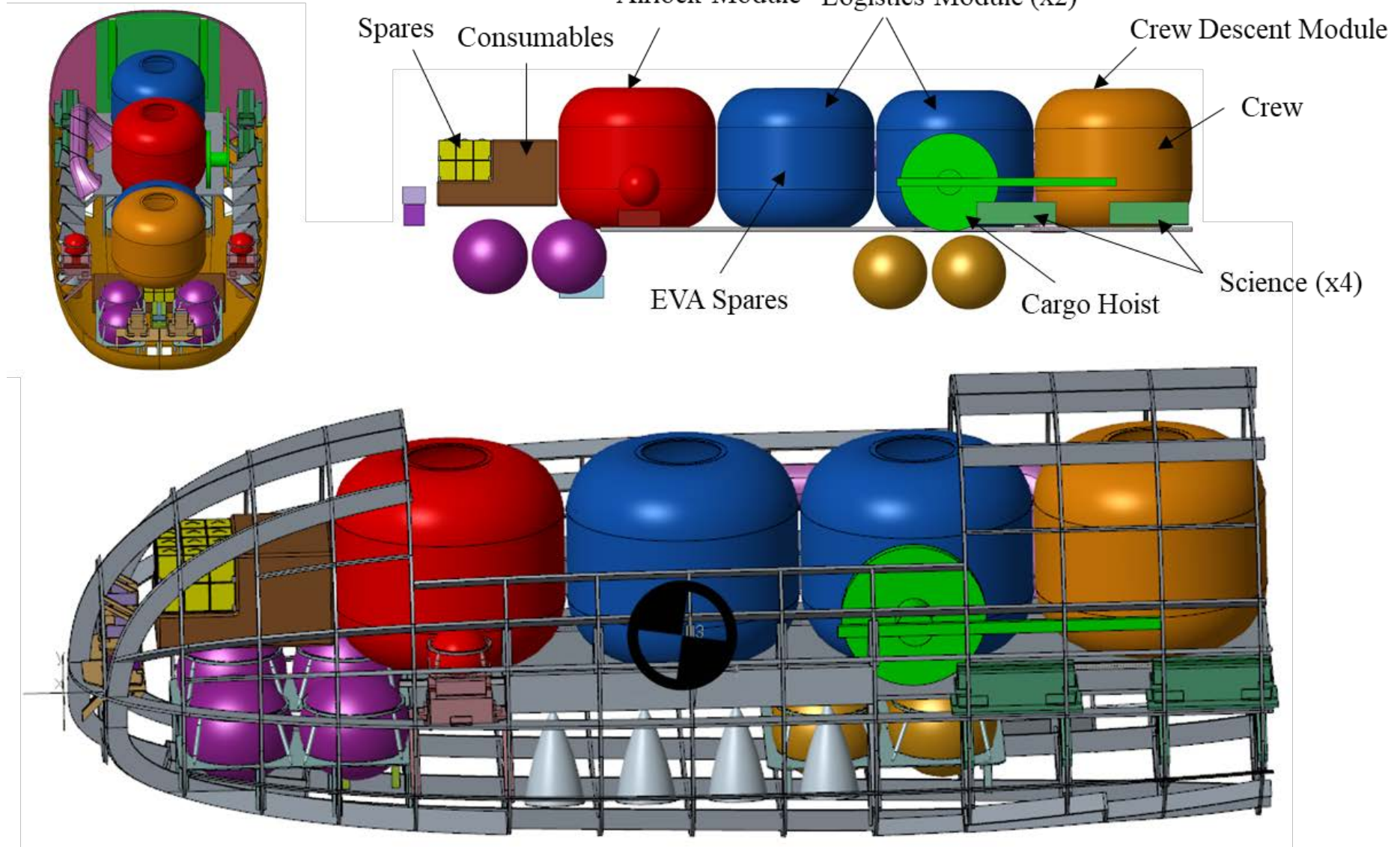


\section{CG Cargo 1}

- Calculated CG with all Cargo-1 items:

- X: $10.557 \mathrm{~m}$

- Y: $\quad-0.0139 \mathrm{~m}$

- Z: $0.393 \mathrm{~m}$
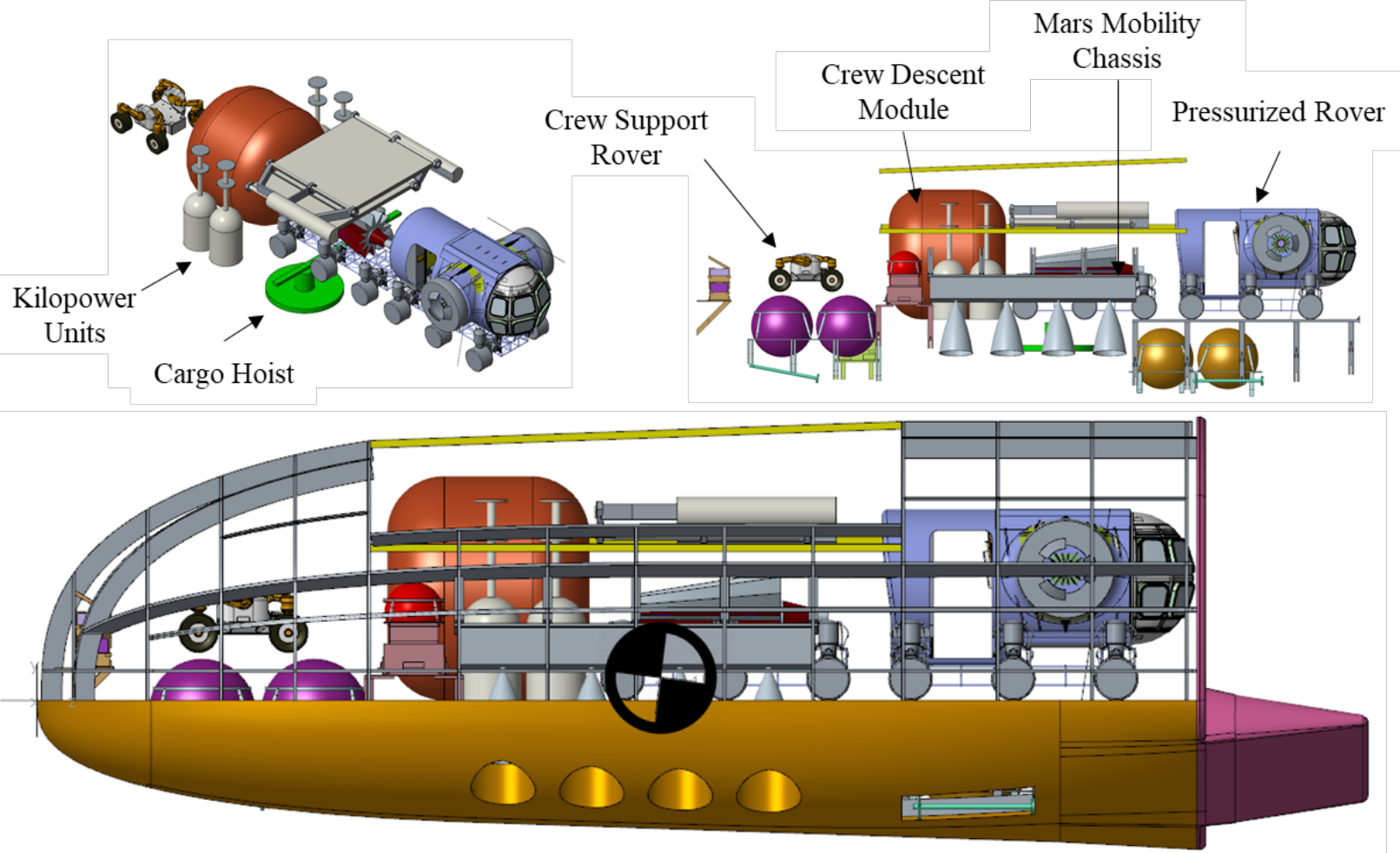


\section{CG Estimations}

- All configurations meet CG requirements

\begin{tabular}{|c|r|r|r|}
\hline Manifest & $\mathbf{X}_{\mathrm{CG}}(\mathbf{m})$ & $\mathbf{Y}_{\mathrm{CG}}(\mathbf{m})$ & \multicolumn{1}{c|}{$\mathbf{Z}_{\mathrm{CG}}(\mathbf{m})$} \\
\hline Cargo-1 & -10.557 & -0.014 & -0.393 \\
\hline Cargo-2 & -10.446 & 0.001 & -1.009 \\
\hline Crew-1 & -10.380 & -0.006 & -0.013 \\
\hline
\end{tabular}


Damien Calderon STRUCTURAL SIZING AND OPTIMIZATION 


\section{Initial Assumptions}

- Used the 2908G OML, manifest packaging for Cargo 2 for sizing

- Modeled frame to match OML form

- Modified substructure to support cargo packaging, dynamic constraints

- MSC Nastran used as a linear solver (SOL 101)

- Collier Research Hypersizer used for sizing optimization

- All sized structure assumes Aluminum 2024 construction 


\section{Load Cases}

Four load cases envelope the design:

1. Earth Launch/Ascent

- +5g Axial, +/-2g Lateral

- 0-0.5 psig Vent Pressure

2. Mars Entry

- Peak Dynamic Pressure

3. Mars Propulsive Descent

- 800 kN

4. Mars Landing

$-3 g$

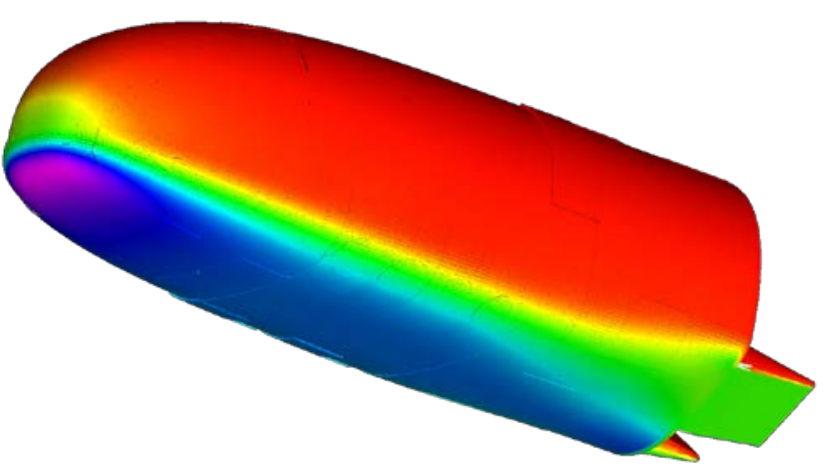




\section{Structural Architecture}

- Skin, Airframe (Ring, Longeron), Substructure

- Components modeled as discrete members, sized independently to allow for optimum mass

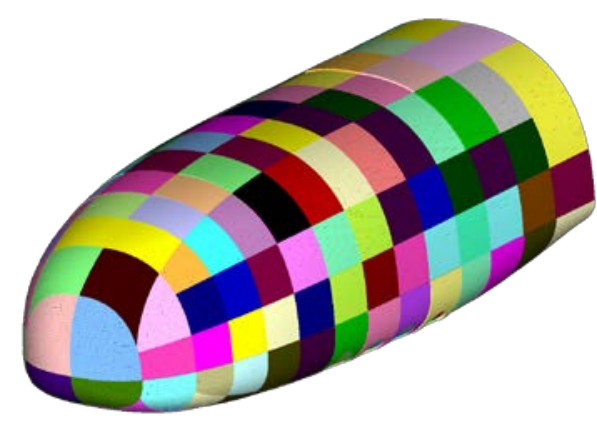

OML Skin

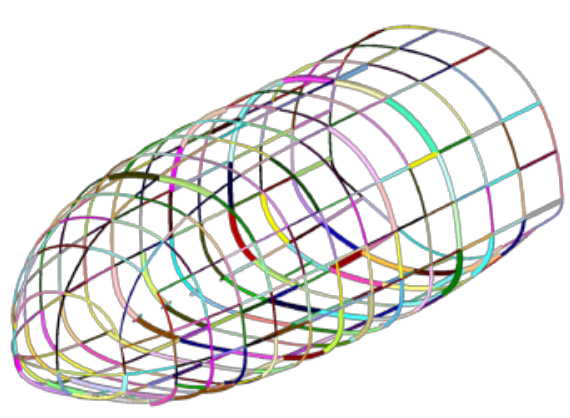

Airframe

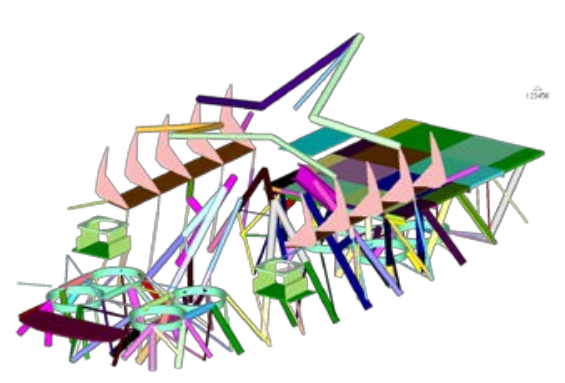

Substructure 


\section{Honeycomb Sandwich Panels}

\section{OML Skin and Cargo Doors}
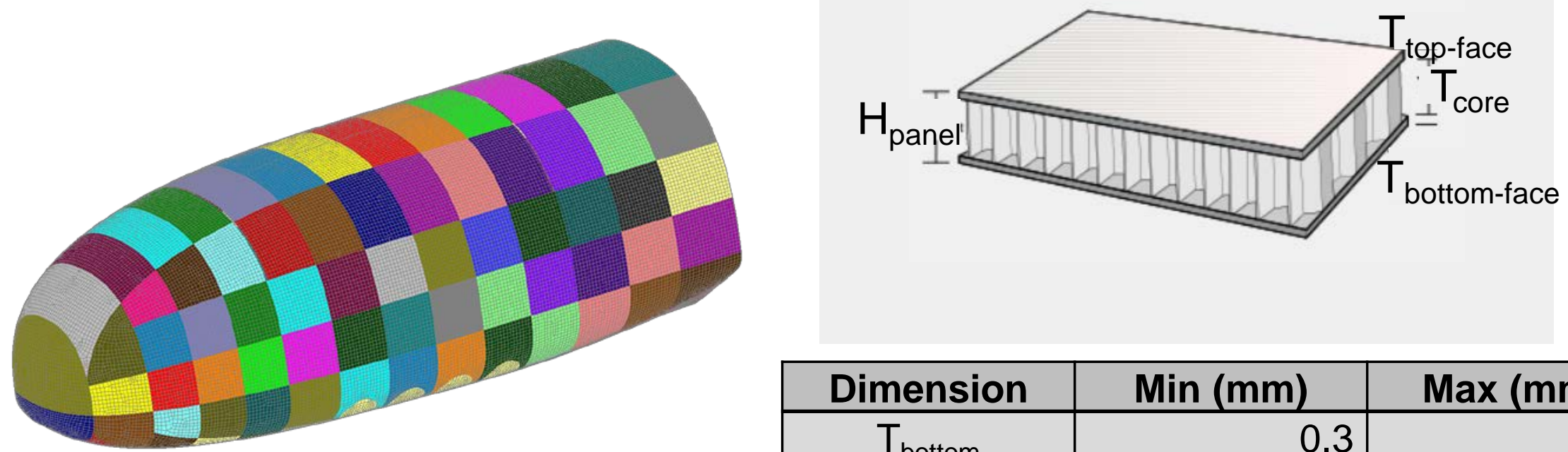

\begin{tabular}{|c|r|r|}
\hline Dimension & \multicolumn{1}{|c|}{ Min (mm) } & \multicolumn{1}{c|}{ Max (mm) } \\
\hline $\mathrm{T}_{\text {bottom }}$ & 0.3 & 3.0 \\
\hline $\mathrm{T}_{\text {core }}$ & 20.0 & 100.0 \\
\hline $\mathrm{T}_{\text {top }}$ & 0.3 & 3.0 \\
\hline
\end{tabular}




\section{Beam Components}

\section{Longerons and Ring Frame}
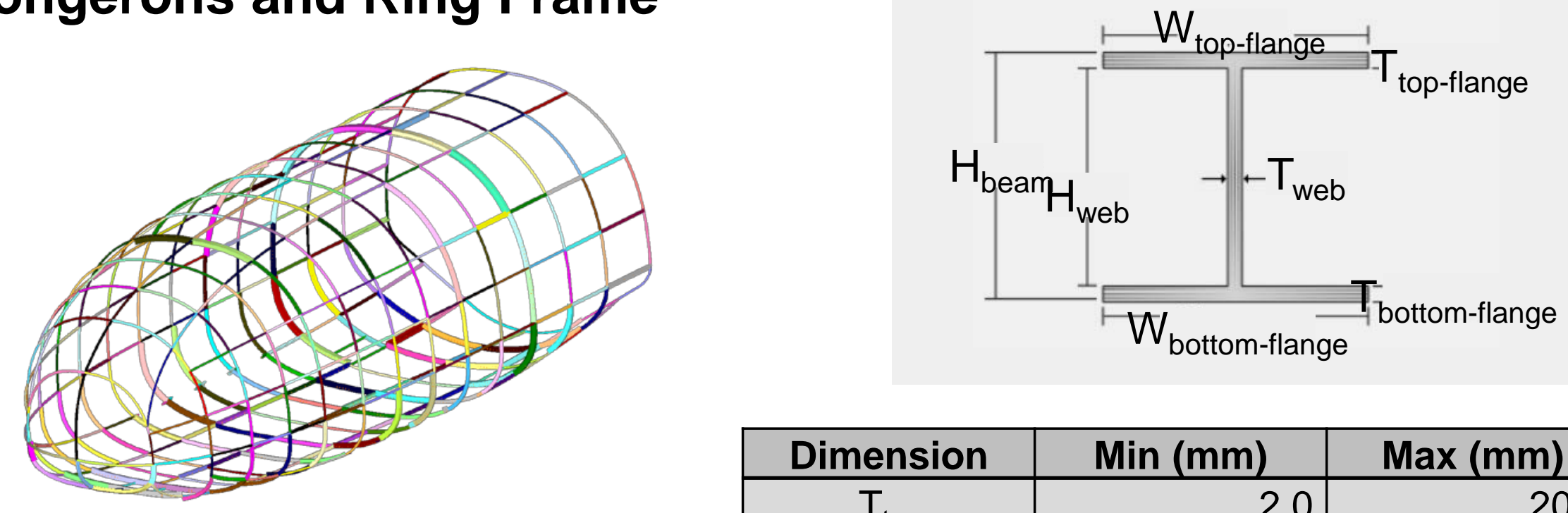

\begin{tabular}{|c|r|r|}
\hline Dimension & \multicolumn{1}{|c|}{ Min (mm) } & Max (mm) \\
\hline $\mathrm{T}_{\text {top }}$ & 2.0 & 20.0 \\
\hline $\mathrm{T}_{\text {web }}$ & 2.0 & 20.0 \\
\hline $\mathrm{T}_{\text {bottom }}$ & 2.0 & 20.0 \\
\hline $\mathrm{W}_{\text {top }}$ & 30.0 & 200.0 \\
\hline $\mathrm{W}_{\text {bottom }}$ & 30.0 & 200.0 \\
\hline $\mathrm{H}_{\text {beam }}$ & 30.0 & 200.0 \\
\hline
\end{tabular}




\section{Beam Components}

\section{Cargo Support Struts}
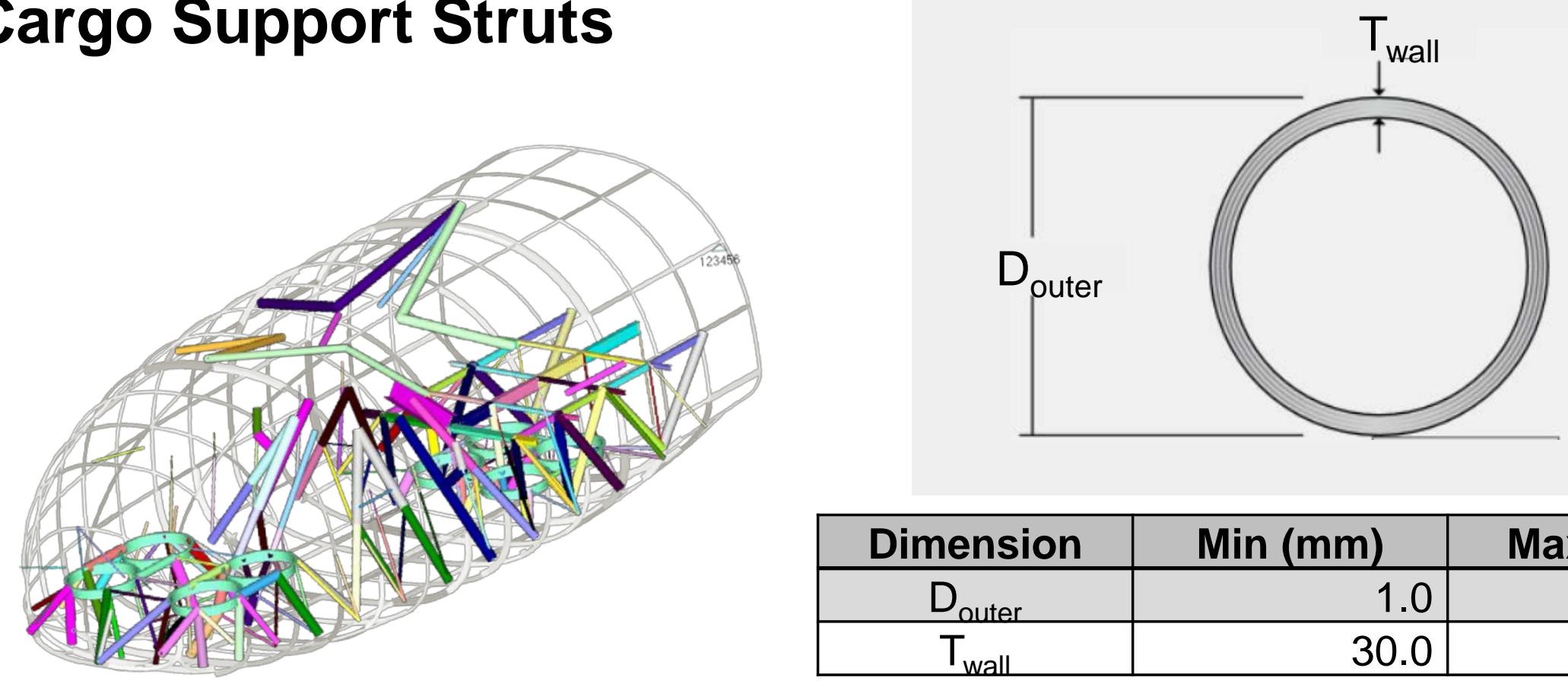

\begin{tabular}{|c|r|r|}
\hline Dimension & \multicolumn{1}{|c|}{ Min (mm) } & Max (mm) \\
\hline $\mathrm{D}_{\text {outer }}$ & 1.0 & 15.0 \\
\hline $\mathrm{T}_{\text {wall }}$ & 30.0 & 250.0 \\
\hline
\end{tabular}




\section{Grid Stiffened Panels}

\section{Substructure}
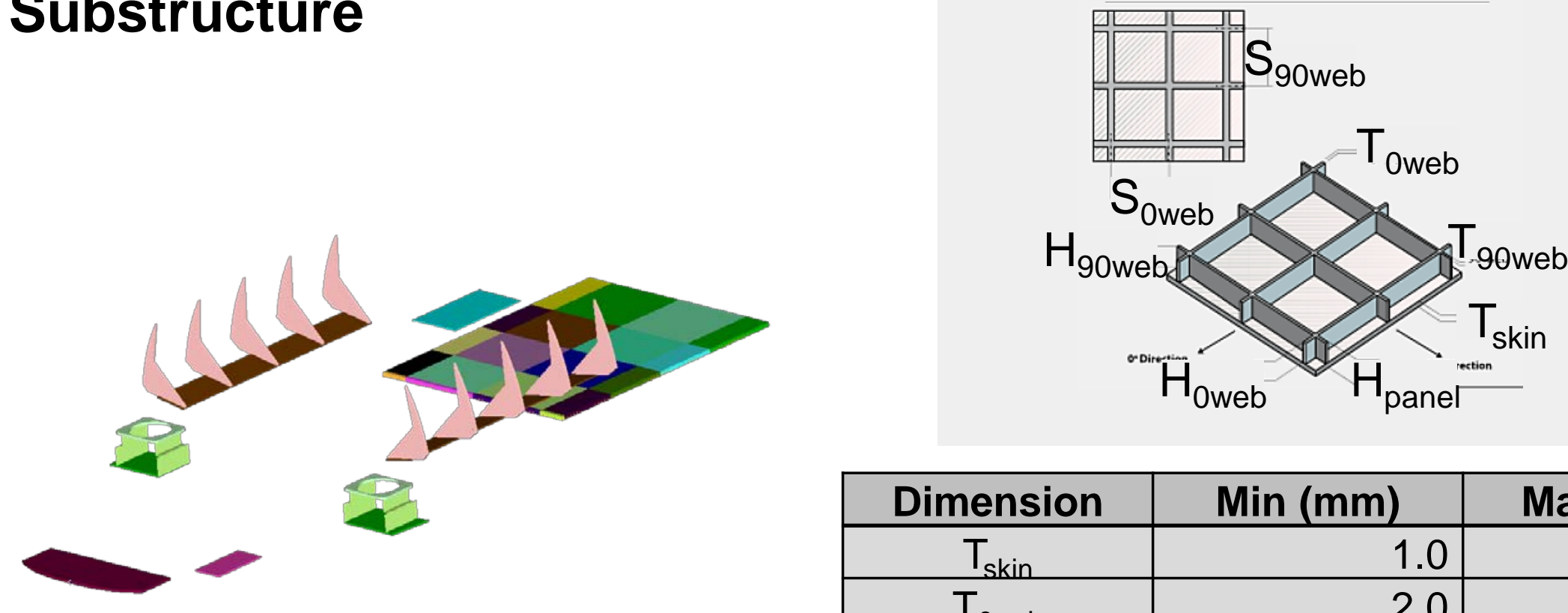

\begin{tabular}{|c|r|r|}
\hline Dimension & \multicolumn{1}{|c|}{ Min (mm) } & Max (mm) \\
\hline $\mathrm{T}_{\text {skin }}$ & 1.0 & 10.0 \\
\hline $\mathrm{T}_{\text {Oweb }}$ & 2.0 & 20.0 \\
\hline $\mathrm{T}_{\text {9oweb }}$ & 2.0 & 20.0 \\
\hline $\mathrm{H}_{\text {oweb }}$ & 20.0 & 100.0 \\
\hline $\mathrm{H}_{\text {goweb }}$ & 20.0 & 100.0 \\
\hline $\mathrm{S}_{\text {Oweb }}$ & 50.0 & 800.0 \\
\hline $\mathrm{S}_{\text {goweb }}$ & 50.0 & 800.0 \\
\hline
\end{tabular}




\section{Basic Mass Results}

\section{Baseline predicted structural mass: 10281 kg}

\begin{tabular}{|c|c|c|c|}
\hline Assembly & Basic Mass (kg) & 20\% MGA (kg) & Predicted Mass (kg) \\
\hline Skin & 4111 & 822 & 4933 \\
\hline Frame & 1483 & 297 & 1780 \\
\hline Substructure & 2973 & 595 & 3568 \\
\hline Total Structural Mass & 8567 & 1714 & 10281 \\
\hline
\end{tabular}


BACKUP 


\section{Cargo Bay Doors (CBD)}

- Orbiter payload bay door (PLBD) system actuator was $0.66 \mathrm{t}$ (1446.2 lbm)

- Assume system mass scales with area PLBD:CBD 1.83:1

- Estimated mass of CobraMRV CBD mechanisms and associated structure is $0.36 \mathrm{t}(793.7 \mathrm{lbm})$
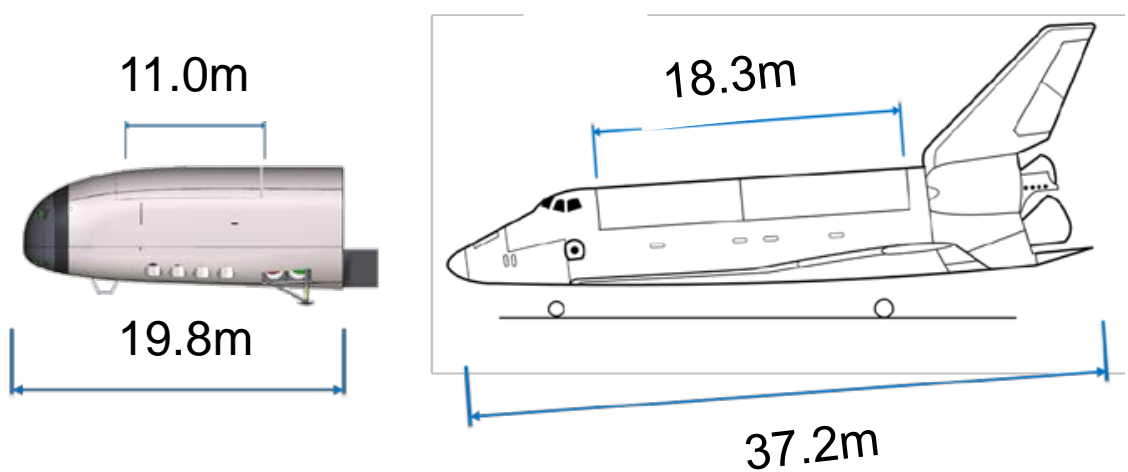

Predicted CobraMRV

\begin{tabular}{|l|l|}
\hline Major Components & \\
\hline Power Drive Units & 10 \\
\hline Rotary Actuators & 8 \\
\hline Latches & 22 \\
\hline Hinges & 16 \\
\hline
\end{tabular}

Total number of parts: 56 


\section{Aft Door/Ramp}

- Assumptions

- Single Use Deployment

- Vent Pressure of $0.5 p s i$

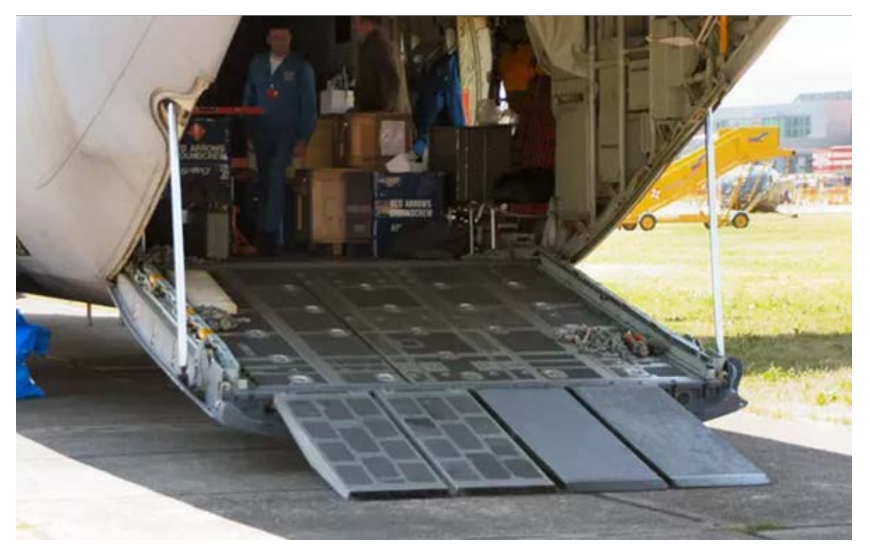

- Design Background - C130 Hercules cargo ramp

- Frangible nuts to secure door, blown upon deployment

- Pin/Hammer system to encourage door deployment

- Struts, hard stop to control drop of door

-Pin system to release support ramp 


\section{Aft Door/Ramp (cont)}

- Estimated door/ramp mass $\sim 177$ kg (389 lbs)

- Estimated mechanisms and seal mass $\sim 209$ kg (460 lbs)
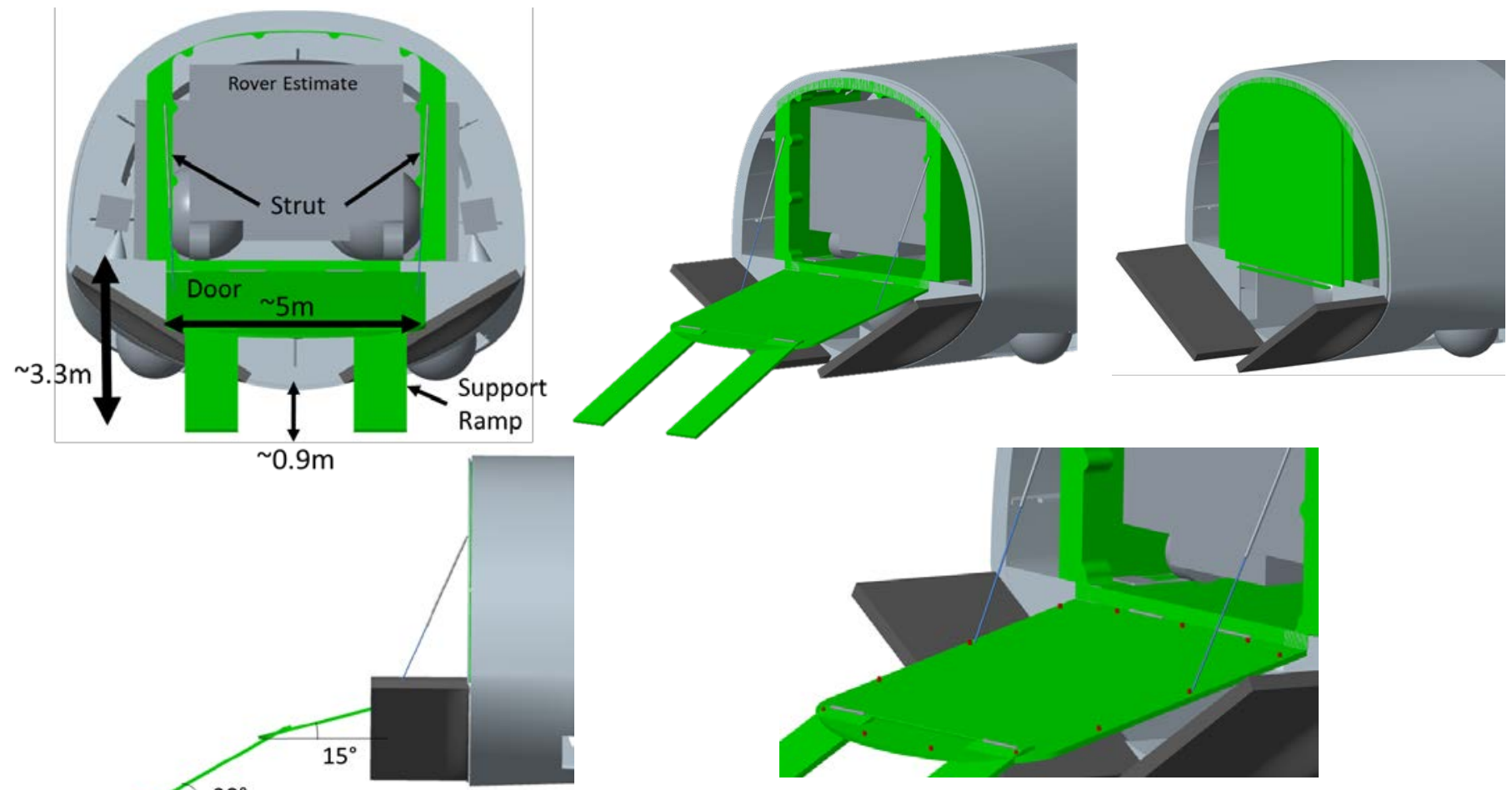